\title{
A Versatile THz Source from High-Brightness Electron Beams: Generation and Characterization
}

\author{
Enrica Chiadroni ${ }^{1, *}\left(\mathbb{D}\right.$, Alessandro Cianchi ${ }^{2}$, Massimo Ferrario ${ }^{1}$, Andrea Mostacci ${ }^{3}$, \\ Riccardo Pompili ${ }^{1}$ (D) and Vladimir Shpakov ${ }^{1}$ \\ 1 Laboratori Nazionali di Frascati (INFN-LNF), Via Enrico Fermi 54, 00044 Frascati, Italy; \\ massimo.ferrario@lnf.infn.it (M.F.); riccardo.pompili@lnf.infn.it (R.P.); vladimir.shpakov@lnf.infn.it (V.S.) \\ 2 University of Rome Tor Vergata and INFN Tor Vergata, Via Ricerca Scientifica 1, 00133 Rome, Italy; \\ alessandro.cianchi@roma2.infn.it \\ 3 Sapienza University of Rome, Department of Basic and Applied Sciences for Engineering, via A. Scarpa 16, \\ 00161 Rome, Italy; andrea.mostacci@uniroma1.it \\ * Correspondence: enrica.chiadroni@lnf.infn.it
}

Received: 24 April 2020; Accepted: 4 June 2020; Published: 8 June 2020

\begin{abstract}
Ultra-short electron bunches, such as those delivered by a high-brightness photo-injector, are suitable to produce high peak power $\mathrm{THz}$ radiation, both broad and narrow band, with sub-picosecond down to femtosecond pulse shaping. The features of this kind of source in the $\mathrm{THz}$ range of the electromagnetic spectrum are extremely appealing for frequency- and time-domain experiments in a wide variety of fields. The present manuscript will overview the method of generation and characterization of $\mathrm{THz}$ radiation produced by high-brightness electron beams, as those available at the SPARC_LAB test facility.
\end{abstract}

Keywords: THz source; high-brightness electron beams; laser comb technique

\section{Introduction}

Terahertz (THz) science [1,2] is recently arousing interest worldwide for its impact in scientific research and technological progress because of the unique characteristics of this radiation. Indeed, $\mathrm{THz}$ radiation is non-ionizing, it penetrates in most insulating materials, e.g., plastic, ceramics, paper, and it is reflected by metals; moreover, it is absorbed by polar liquids, showing absorption spectra peculiar of the physical process. As a matter of fact, powerful $\mathrm{THz}$ sources are highly demanded, since they can provide efficient tools to investigate matter in the non-linear regime. Moreover, both biomedical and security applications can profit from the development of innovative $\mathrm{THz}$ sources, detection devices and characterization techniques.

$\mathrm{THz}$ radiation lies between the photonics and electronics bands of the electromagnetic spectrum. Effectively, this frequency region extends from $300 \mathrm{GHz}$ up to $30 \mathrm{THz}$, corresponding to energies, in the meV range, of great interest for many physical, chemical and biological processes. Furthermore, molecular spectroscopy and imaging [3] can take advantage of a high peak power, tunable and narrow-band $\mathrm{THz}$ radiation.

Unlike conventional THz sources, such as those based on diodes, a new generation of sources, based on particle accelerators, allows a raising of the power, both average and peak, by many orders of magnitude. In particular, linac-based coherent $\mathrm{THz}$ radiation from relativistic high-brightness electron beams is a cutting-edge source [4-6] providing an efficient tool to investigate matter in the non-linear regime. Indeed, if the electron bunch longitudinal structure does not change during the emission process, the $\mathrm{THz}$ radiation is characterized by pulses whose temporal shape resembles the electron bunch one and with associated peak electric fields greater than 1-10 MV/cm [7]. 
The great advantage of a linac-based $\mathrm{THz}$ source lies in its versatility; indeed, the electron beam longitudinal distribution can be properly shaped to generate tunable, few-cycle and multi-cycle, coherent $\mathrm{THz}$ radiation with both broad and narrow spectral bandwidth. In particular, to enable the generation of narrow-band $\mathrm{THz}$ radiation, different approaches that foresee the longitudinal manipulation of the electron beam are currently used: temporal shaping of the photocathode drive laser pulse [8-11], mask insertion in a high dispersion, low beta function region of a beamline dogleg [12], transverse-to-longitudinal phase space exchange technique [13] to turn transverse modulation from a multi-slit into a longitudinal one, and magnetic undulator radiation [14-19]. Here, we report on the first approach, being the one developed and fully studied at the SPARC_LAB test facility [20].

\section{Materials and Methods}

The key for high efficiency in a beam-based radiation source is to exploit the coherence enhancement effect boosted by tailoring the electron beam profile. For instance, coherent radiation in the $\mathrm{THz}$ range can be generated through ultra-short, i.e., sub-ps, relativistic electron bunches hitting a metal target as Transition Radiation (TR) [21]. Indeed, when a charged particle crosses the interface between two different refraction index media, TR is emitted both in forward and backward direction. The radiation results from the prompt change of the boundary conditions for the electromagnetic (EM) field carried by the particle in the first and second media. When a charged particle goes through a slit, opened in a metal target, only part of the particle field interacts with the screen, and the emitted radiation is called Diffraction Radiation (DR). Since the particle goes through the aperture without interacting with the screen, the emission process is not intercepting and non-invasive for the particle itself. Coherent Diffraction Radiation (CDR) is particularly appealing as non-disruptive longitudinal diagnostics [22].

When a bunch of $N$ charged particles is considered, TR (DR) emission comes from each particle with the same intensity, but the position of each particle in the bunch has an impact on the emission, contributing with a phase term. Therefore, two regimes can be identified depending on the bunch length, $\sigma_{z}$, with respect to the emission wavelength, $\lambda$ : at $\lambda \ll \sigma_{z}$, each particle behaves independently from each other, resulting in incoherent emission, proportional to the number of electrons in the bunch, $N$; at $\lambda \gg \sigma_{z}$, all electrons emit in phase and the amplitudes add constructively, resulting in coherent emission, proportional to $N^{2}$. Therefore, the total emitted energy can be written as

$$
\frac{d U}{d \lambda}=\frac{d U_{s p}}{d \lambda}\left[N+N(N-1)|F(\lambda)|^{2}\right] .
$$

$F(\lambda)$ is the longitudinal bunch form factor, defined as $F(\lambda)=\int_{-\infty}^{\infty} S(z) e^{-2 \pi i z / \lambda} d z$, with $S(z)=$ $\int S_{3 D}(\vec{r}) d x d y$ the longitudinal charge distribution, i.e., the projection of the three-dimensional normalized particle density distribution onto the $\mathrm{z}$ axis. The bunch longitudinal form factor sets the emission regime: at wavelengths longer than the bunch length, $F(\lambda) \rightarrow 1$ and the spectrum is dominated by coherent emission, which extends to $\mathrm{THz}$ frequencies for sub-ps electron beams, with a huge intensity gain with respect to most existing THz sources thanks to the $N^{2}$ dependency.

\subsection{Broad Band Radiation}

Short, i.e., sub-ps, electron bunches drive the generation of high peak power radiation in the $\mathrm{THz}$ range. In linac-based facilities short electron bunches are typically produced by either magnetic or radio-frequency (RF) compression techniques. Magnetic compression, performed at high energy to overcome space charge effects, foresees a bunch with a time-energy correlation (or chirp) moving along an energy-dependent path length, represented by a dispersive, non-isochronous beam transport section. As alternative at lower energies, in the few $\mathrm{MeV}$ range, the RF compression scheme, known as velocity bunching [23] (VB), exploits the interaction with the electromagnetic fields of an accelerating cavity. A time-velocity correlation (or chirp) is provided to the electron bunch, inducing a longitudinal phase space rotation in the traveling RF wave potential due to the fact that electrons in the bunch tail are 
faster than those in the bunch head. Since the beam is slightly relativistic, being slower than the phase velocity of the RF wave, when injected at the zero crossing field phase, it slips back to phases where the field is accelerating and, simultaneously, it is chirped and compressed. Therefore, compression and acceleration take place at the same time within the same accelerating section. Beyond the longitudinal dimension, the electron beam longitudinal shape also plays a significant role in the spectral content of the emission. In particular, asymmetric current profiles, caused by both longitudinal and transverse distortions of the phase space, and typical of compression techniques, promote emission at higher $\mathrm{THz}$ frequencies. Indeed, Figure 1 shows the longitudinal form factor as function of the frequency for four different beam longitudinal distributions, with the same RMS pulse duration (inset): Gaussian (black line), uniform (red line), triangular (blue line), and real measured one (green asterisks). The triangular-like longitudinal distribution results as the most efficient one to generate $\mathrm{THz}$ radiation, thanks to the steep rising edge, which is also shown in the longitudinally compressed beams (see green asterisks curve in the inset of Figure 1).

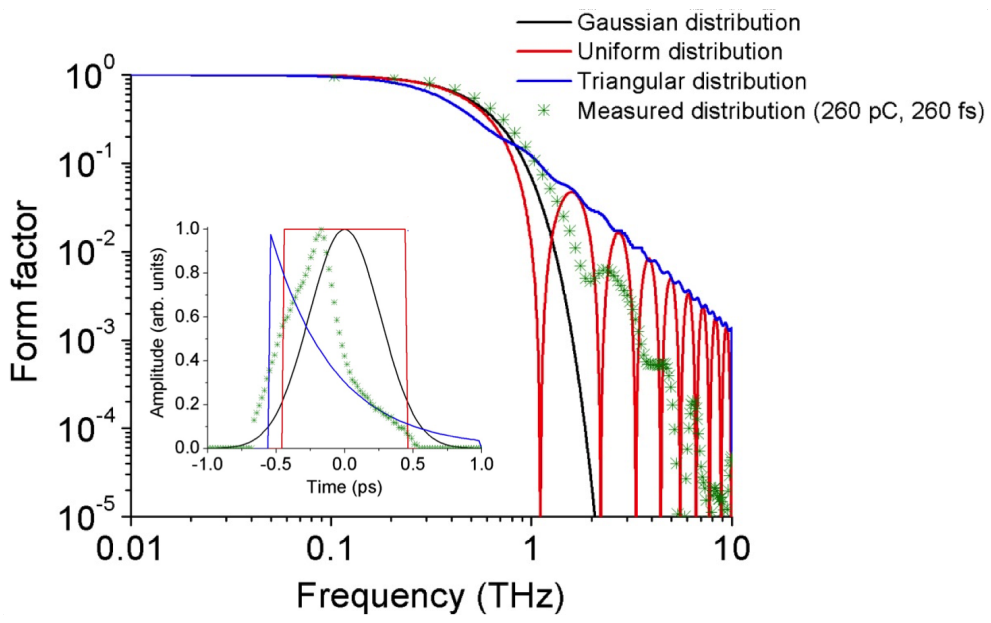

Figure 1. Longitudinal bunch profile (inset) and corresponding form factors for three different particle distribution with the same RMS pulse duration, i.e., 260 fs.

\subsection{Narrow-Band and Tunable Radiation}

One of the methods used to produce narrow-band and tunable $\mathrm{THz}$ radiation is based on the generation of Coherent Transition Radiation (CTR) from a train of sub-ps electron bunches hitting a metal target $[10,24]$.

The generation of a train of sub-picosecond electron bunches with picosecond spacing has been conceived and demonstrated at the SPARC_LAB test facility [25], applying the VB compression technique to a longitudinally modulated electron bunch as generated by a train of UV laser pulses hitting the photocathode, the so-called comb laser beam [26].

One way to generate comb laser pulses relies on birefringent crystals: an input laser pulse is decomposed into two orthogonally polarized pulses with a time separation proportional to the crystal length. A multi-peak laser profile is produced by stacking more birefringent crystals in the laser beam path. The so-generated comb laser pulse, illuminating a metallic photocathode in a RF gun, produces a train of short electron bunches thanks to the prompt temporal response of the metal, which allows for transverse and longitudinal shaping of the electron beam through laser manipulation. Downstream the photo-injector, the beam acquires an energy modulation because of the space charge forces and, if injected in a RF-compressor operating in the over-compression regime, the energy modulation turns back into density modulation. The train parameters, i.e., bunch charge, length, and time separation, can be fully controlled by properly adjust both laser and linac settings [27]. A train of sub-ps relativistic electron bunches intercepting an aluminum screen, emits CTR. Since the CTR emission is a surface phenomenon, it happens with femtosecond timescale and the electron bunch structure 
does not change during the emission process; in addition, no interaction occurs between particles and radiation, resulting in a radiation pulse structure that resembles the multi-bunch longitudinal one with a spike-like spectrum at the frequency of the bunch distance and higher harmonics, as depicted in Figure 2.
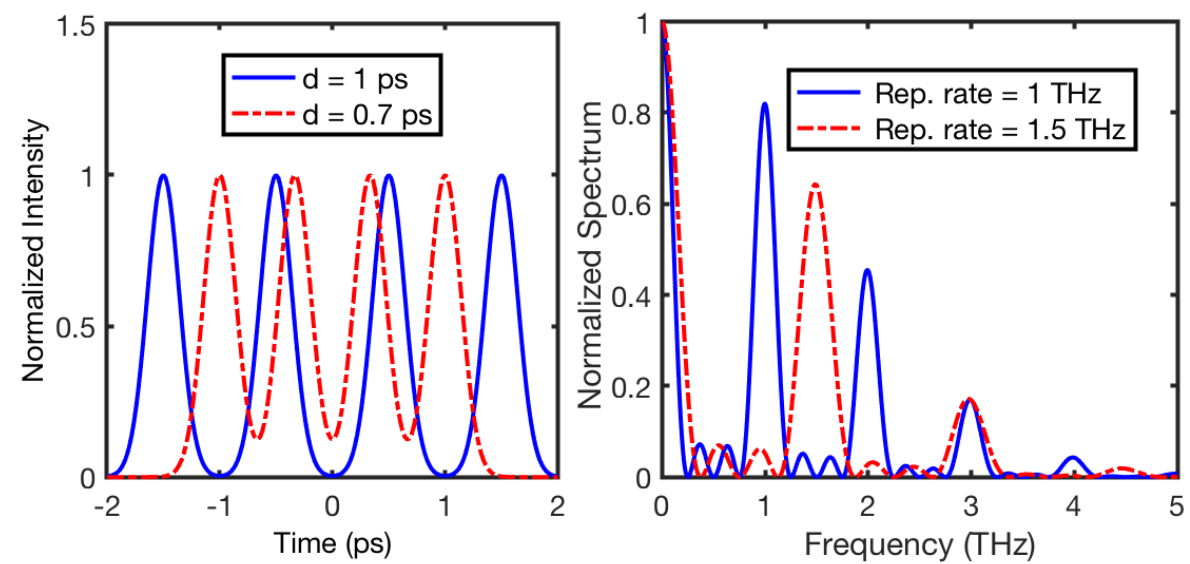

Figure 2. Left: 4-bunches comb beam with different time separation (blue solid line, 1 ps; red dashed line, $0.7 \mathrm{ps}$ ). Right: Corresponding form factors highlighting the tunability of the narrow-band $\mathrm{THz}$ source.

Decreasing the sub-pulse length, the single pulse spectrum becomes larger and more harmonics of the sub-pulse repetition frequency appears in the comb spectrum, with the advantage that the energy at single frequency is the same as all the charge would be condensed in a single micro-pulse. In addition, the central $\mathrm{THz}$ frequency, and its harmonics, can be tuned by changing the micro-bunches time separation.

\subsection{Experimental Layout}

CTR is generated, at the SPARC_LAB test facility, by highly relativistic electron beams hitting a $40 \times 40 \mathrm{~mm}$, aluminum coated silicon screen (380 $\mu \mathrm{m}$ thick with $40 \mathrm{~nm}$ coating). A proper optical system is needed to extract the CTR and transport it down to the detection apparatus and, eventually, to the sample. A sketch of the experimental layout, with light optics showing the radiation path, is depicted in Figure 3. Radiation is extracted from the vacuum pipe through a crystalline diamond window (700 $\mu \mathrm{m}$ thick, $0.3 \mathrm{deg}$ wedge, $40 \mathrm{~mm}$ clear aperture), and collected by aluminum coated parabolic mirrors.

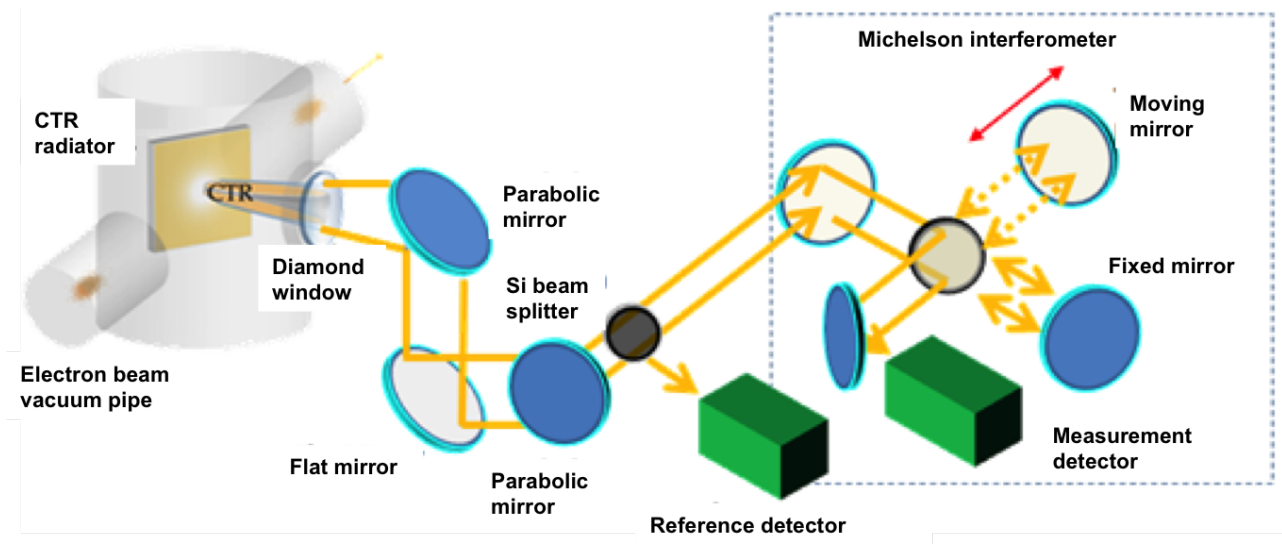

Figure 3. Experimental layout. 
The coherent $\mathrm{THz}$ source is currently characterized by measuring the radiation spectrum in air (or dry air environment, i.e., $12 \%$ of relative humidity at $297 \mathrm{~K}$ ) both through a set of discrete band-pass $\mathrm{THz}$ filters and an interferometer. The dry air system, a Kaeser Membrane Module dryer (FE-6D D-pack) working with compressed air, contributes to reduce water absorption in the Perspex box embedding the experimental apparatus (overall volume of about 200 litres), allowing measurement of radiation from very low charge bunches, of the order of $10 \mathrm{pC}$, needed for the production of ultra-short, fs-scale, electron bunches.

A two-beam interferometer is usually used for frequency domain measurements. For ps and sub-ps electron bunches a Martin-Puplett interferometer (MPI) [28] is preferred because of the beam splitter properties. Indeed, despite a Michelson interferometer, the beam splitter is a wire grid whose reflectivity coefficients depend on wires diameter and spacing. Furthermore, it splits polarizations, thus measuring the intensity of both polarizations: the difference interferogram can be normalized to the sum. Correlated fluctuations due to electron beam current instabilities are then canceled. The main limitation is however due to the high-frequency suppression introduced by the spacing between wires, which prevents the measurement of ultra-short bunches. Therefore, for bunches whose duration is from a few hundreds of fs down to a few tens of $\mathrm{fs}$, the most suitable choice is a Michelson interferometer, which allows the covering of a wide spectral range, from 0.5 to $30 \mathrm{THz}$, by properly changing the thickness of the beam splitter from $24 \mu \mathrm{m}$ down to $3.5 \mu \mathrm{m}$.

The autocorrelation measurements reported here have been performed using a Michelson interferometer with an additional detector (reference detector in Figure 3), which collects the radiation after a silicon beam splitter, placed before the Michelson interferometer box. The intensity measured by the measurement detector is normalized to the one measured by the reference detector, contributing to an increase of the signal to noise ratio; as a matter of fact this upgrade to the conventional Michelson interferometer allows the cancelation of correlated fluctuations due to electron beam current instabilities as in the two-beam MPI.

\section{Results and Discussion}

The versatility of linac-based THz sources stands in the possibility to tune the $\mathrm{THz}$ spectral content by properly setting the photo-injector regime and the UV photocathode laser longitudinal distribution for either narrow or broad band $\mathrm{THz}$ emission. In case of a longitudinally modulated electron beam, whose current profile and longitudinal phase space are shown in Figure 4 (left and right, respectively), the measured interferogram (Figure 5, left) presents the peculiar multi-peaks pattern, in particular $(2 n-1)$ peaks, with $n=5$ the number of bunches in the train. At zero optical path difference each sub-pulse within the two comb-like beamlet completely overlaps, resulting in the central maximum in the interferogram. As the path difference increases, the interferogram intensity decreases until four of the five pulses overlap, corresponding to the first side maxima. Further increasing the distance, the interferogram intensity keeps on decreasing resulting in the farther side maxima.

The spectrum corresponding to the autocorrelation function of the radiation produced by the 5 bunches beam is shown in Figure 5 (right). The main peak is at $0.65 \mathrm{THz}$ corresponding to a $1.54 \mathrm{ps}$ time separation between two of the five bunches, with a bandwidth (BW) of around $30 \%$, whereas commercial THz filters have around $15-20 \%$ BW. Since the single bunch length in the train is of the order of $100 \mathrm{fs}$ and less, a second and third harmonics arouse at around $1.25 \mathrm{THz}$ and $1.95 \mathrm{THz}$. The peak at around $1 \mathrm{THz}$ corresponds to the $0.8-0.9$ ps distance of two of the four bunches, as shown in the electron beam current profile and longitudinal phase space.

Therefore, paying careful attention to a regular stacking of similar electron bunches, produces the suppression of all the frequencies apart the one corresponding to the succession itself, reaching in intensity at most the value of the single sub-pulse case. It is worth clarifying that to obtain the same radiation intensity at this frequency, in the case of a single bunch all the electrons $N$ must be concentrated in the single bunch itself, with the drawback that space charge usually prevents to collect high charge in $<100 \mathrm{fs}$. The width of this line depends on the number of pulses that constitutes the 
comb. Therefore the radiation produced by a longitudinally modulated beam (comb beam) is emitted at the frequency of comb and higher harmonics depending on the sub-bunch length, and the intensity at those frequencies is as if all electrons $N$ would be concentrated in the single bunch whose duration is of the order of $100 \mathrm{fs}$ or less. The transform limit is not violated, since the overall bandwidth is wide due to the fact that not only the fundamental frequency is populated, but also the higher harmonics. In addition, the shorter the pulse, the wider is the THz spectrum, because more harmonics show up. As a matter of fact, the photons of the total bandwidth are distributed differently, based on the temporal structure of the bunch, as its Fourier transform shows (Figure 5, right). Therefore the total peak power must be evaluated assuming that all the charge is collected in the micro-bunch duration, since it is the duration of the single micro-bunch in the train that sets the extension of the spectrum, thus the number of harmonics. Therefore, the term narrow for the frequency bandwidth of radiation from a train of micro-bunches refers to the bandwidth of each line of the frequency spectrum, which is naturally filtered from both the number and duration of bunches in the train.
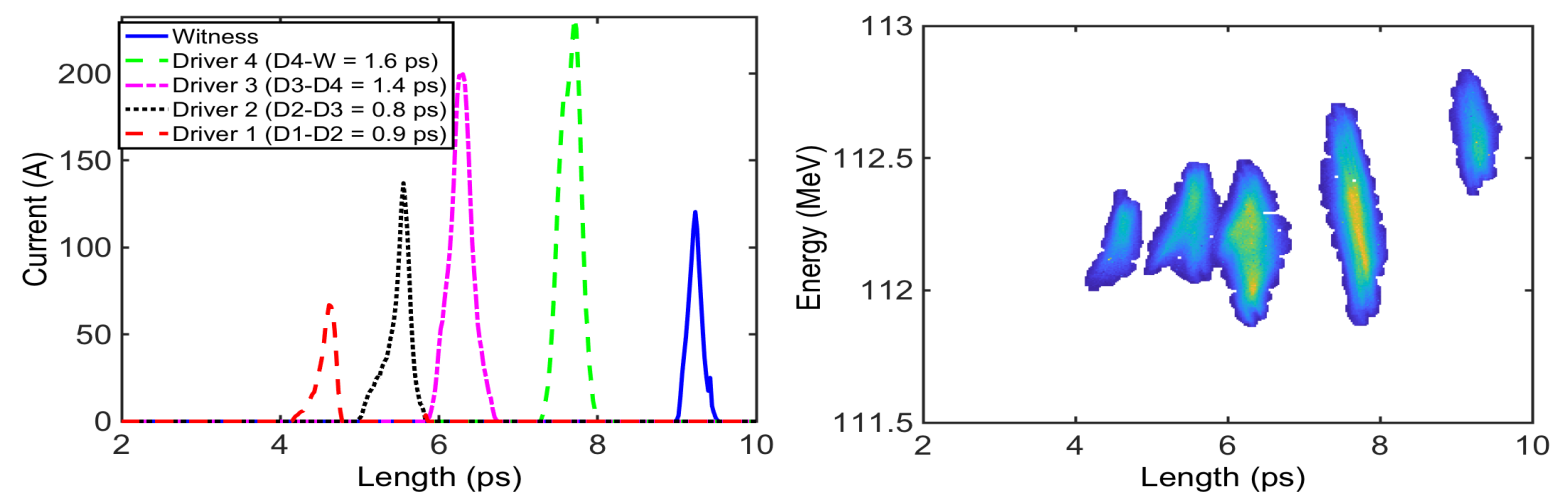

Figure 4. Electron beam longitudinal profile (left) and longitudinal phase space of a 5-bunches ramp train (right).
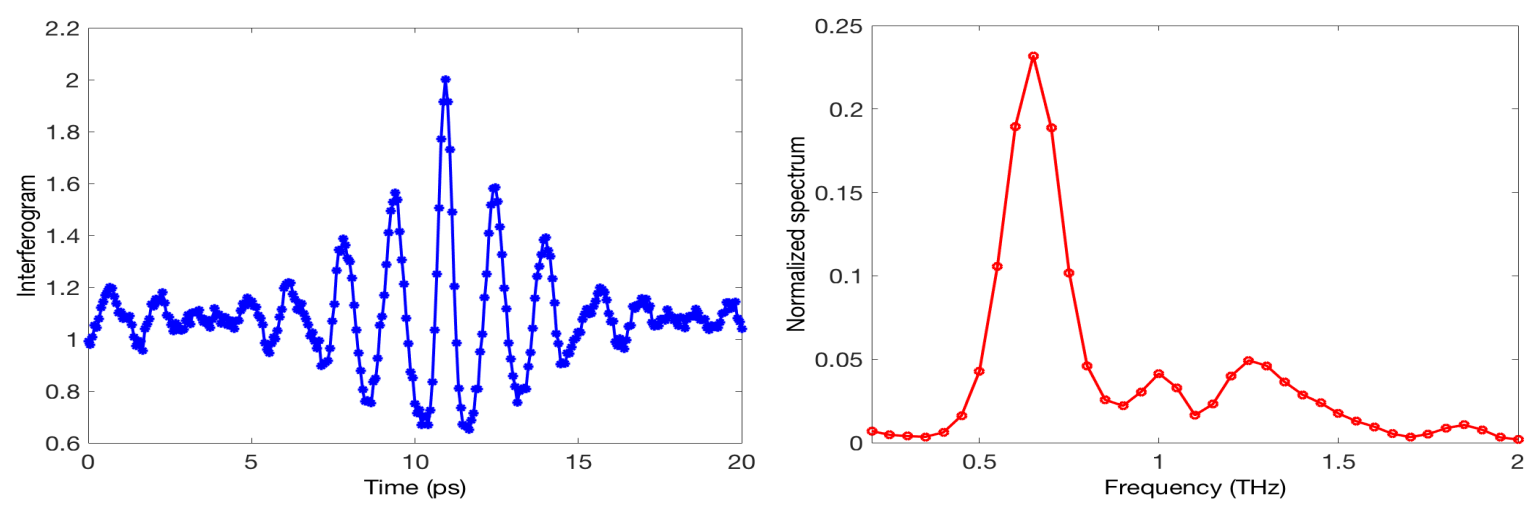

Figure 5. Autocorrelation function (left) and relative spectrum (right) of a 5-pulses generated radiation.

The spectral properties of the $\mathrm{THz}$ radiation, as generated by a single sub-ps electron bunch can be also investigated sampling the signal by means of commercial band-pass THz filters [29]. Highly intense and broadband CTR has been produced, for instance, by a $110 \mathrm{MeV}, 500$ pC, 180 fs RMS beam impinging on an aluminum coated silicon screen, and its spectrum measured up to $5 \mathrm{THz}$, as shown in Figure 6. 


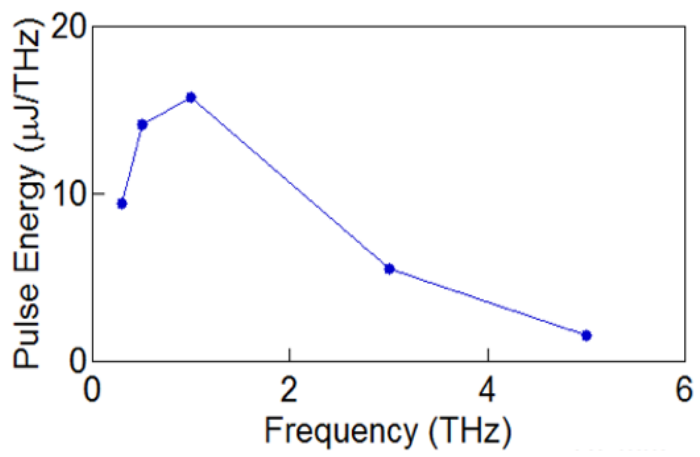

Figure 6. Measured CTR spectrum sampled with THz band-pass filters center at 0.3-0.5-1-3-5 THz [11].

The drop below $0.3 \mathrm{THz}$ in Figure 6 is due to the effect of the screen finite size with respect to the source size [30], being of the order of $\gamma \lambda / 2 \pi$ ( $\gamma$ the Lorentz factor and $\lambda$ the emitted wavelength), combined with the fall of responsivity of the pyroelectric detector. On the other hand, the longitudinal extension of the bunch limits the generation above $5 \mathrm{THz}$. The integrated energy per pulse reaches up to $35 \mathrm{uJ}$ as measured by a pyroelectric detector, whose responsivity and absorption curves have been provided by manufacturers. The pyrodetector is a $5 \mathrm{~mm}$ diameter THz-I-BNC GENTEC-EO, operating in the spectral range $0.1-30 \mathrm{THz}$, with responsivity $\approx 140 \mathrm{kV} / \mathrm{W}$ and a Noise Equivalent Power at $20 \mathrm{~Hz} \approx 4 \times 10^{-10} \mathrm{~W} / \mathrm{Hz}^{0.5}$.

The main figures of merit of the SPARC_LAB THz source for a single bunch to generate broadband $\mathrm{THz}$ radiation and five-ramp comb train to generate a narrow-band $\mathrm{THz}$ radiation are reported in Table 1 [11].

Table 1. SPARC_LAB THz sources: radiation and electron beam parameters for a single bunch and a ramped five-bunch train $\left(\sigma_{t}(D 1)=88 \mathrm{fs}, \sigma_{t}(D 2)=127 \mathrm{fs}, \sigma_{t}(D 3)=151 \mathrm{fs}, \sigma_{t}(D 4)=165 \mathrm{fs}\right.$, $\left.\sigma_{t}(W)=100 \mathrm{fs}\right)$.

\begin{tabular}{cccccc}
\hline \multicolumn{3}{c}{ THz Radiation Parameters } & \multicolumn{2}{c}{ Electron Beam Parameters } \\
\hline & Single Bunch & $\begin{array}{c}\text { Ramped } \\
\text { Comb }\end{array}$ & & Single Bunch & $\begin{array}{c}\text { Ramped } \\
\text { Comb }\end{array}$ \\
\hline Energy per pulse $(\mu \mathrm{J})$ & $35^{+}$ & $\sim 1$ & Charge $(\mathrm{pC})$ & 500 & 220 \\
Peak power $(\mathrm{MW})$ & $\sim 80^{\dagger}$ & $\sim 3$ & Energy $(\mathrm{MeV})$ & 121 & 110 \\
Electric field $(\mathrm{MV} / \mathrm{cm})$ & $>1^{+}$ & - & RMS Bunch duration $(\mathrm{fs})$ & 180 & ${ }^{+}$ \\
Bandwidth $(\mathrm{THz}) \Delta v$ & $\sim 2$ & 0.25 & Rep. Rate $(\mathrm{Hz})$ & 10 & 10 \\
RMS Pulse duration $\mathrm{t}_{p}(\mathrm{ps})$ & $\sim 0.18$ & $\sim 1.23^{* *}$ & Comb distance $(\mathrm{ps})$ & - & 1.3 \\
\hline
\end{tabular}

${ }^{+}$Systematic uncertainty due to missing detector calibration below $0.61 \mathrm{THz} ;{ }^{+\dagger}$ the RMS duration, $\sigma_{t}$, for each bunch in the train is reported in the table caption; * Defined as the FWHM; ${ }^{* *}$ From measured results the time-bandwidth product is $\Delta v \mathrm{t}_{p}=0.72$.

Linac-based THz sources are characterized by pulse energies reaching the multi-hundreds $\mu \mathrm{J}$ regime [31] by increasing the charge, thanks to the $N^{2}$ dependence, and/or properly shaping the bunch longitudinal profile. In addition, taking profit of the beam longitudinal manipulation, the spectral bandwidth can be either tunable narrow or broad, making it extremely versatile. Finally, the repetition rate can span from a few $\mathrm{Hz}$ up to $\mathrm{GHz}$, depending on the accelerator repetition rate, paving the way to an average power of the order of $10 \mathrm{~W}$ [16]. On the other hand, laser-driven $\mathrm{THz}$ sources, in particular those based on Optical Rectification [32] with organic crystals, have demonstrated to achieve hundred $\mu \mathrm{J}$ pulses in tight focus resulting in electric and magnetic field larger than $10 \mathrm{MV} / \mathrm{cm}$ and 3 Tesla, over a broad $0.1-8 \mathrm{THz}$ frequency range [33].

Compared to other linac-based sources [16], and based on the measured parameters, listed in Table 1, the SPARC_LAB THz radiation is strongly competitive and extremely suitable for investigating non-linear THz spectroscopy and for testing novel detectors and materials. Indeed, thanks to $\mathrm{MV} / \mathrm{cm}$ electric fields, which can excite fundamental quantum statuses of a given system, it allows for non-linear optic experiments [34]. 


\section{Conclusions}

In photo-injector-based facilities, $\mathrm{THz}$ radiation can be produced under different regimes, by a proper control of the bunch shaping and charge distribution. Indeed, taking profit of the prompt photoemission of electrons at the metal cathode, different electron longitudinal distribution can be tailored and manipulated by properly setting the UV photocathode laser and the photo-injector parameters.

In particular, the present manuscript reports the most valuable results obtained at SPARC_LAB with both ultra-short single bunch and multi-bunches electron comb beams to provide high energy per pulse and broad and narrow spectral bandwidth $\mathrm{THz}$ radiation, respectively for non-linear and pump-probe experiments in solid-state physics and material science.

Author Contributions: E.C. planned and managed the THz activities at SPARC_LAB, and wrote the manuscript; V.S. was involved in the project and setup of the experimental apparatus; M.F. coordinated the SPARC_LAB test facility; A.C. took part to the preparation of the experiments; A.M. and E.C. carried out the data analysis; R.P. provided numerical simulations for photo-injector settings. All authors were involved in the operation of the experiments, extensively discussed the results and reviewed the manuscript. All authors have read and agreed to the published version of the manuscript.

Funding: This research was partially funded by CSN5 of INFN.

Acknowledgments: Authors would like to acknowledge all the colleagues at the SPARC_LAB test facility of the INFN Frascati and colleagues within the collaboration, in particular, S. Lupi (Sapienza University of Rome) and F. Giorgianni (PSI).

Conflicts of Interest: The authors declare no conflict of interest.

\section{References and Note}

1. Dhillon, S.S.; Vitiello, M.S.; Linfield, E.H.; Davies, A.G.; Hoffmann, M.C.; Booske, J.; Paoloni, C.; Gensch, M.; Weightman, P.; Williams, G.P.; et al. The 2017 terahertz science and technology roadmap. J. Phys. D: Appl. Phys. 2017, 50, 043001. [CrossRef]

2. Lee, Y.-S. Principles of THz Science and Technology; Springer Science+Business Media, LLC: New York, NY, USA, 2009; ISBN 978-0-387-09539-4.

3. Sherwin, M.S.; Schmuttenmaer, C.A.; Bucksbaum, P.H. Opportunities in THz Science. In Proceedings of the Report of a DOE-NSF-NIH Workshop, Arlington, VA, USA, 12-14 February 2004.

4. Hoffmann, M.C.; Schulz, S.; Wesch, S.; Wunderlich, S.; Cavalleri, A.; Schimdt, B. Coherent single-cycle pulses with $\mathrm{MV} / \mathrm{cm}$ field strengths from a relativistic transition radiation light source. Opt. Lett. 2011, 36, 4473-4475. [CrossRef] [PubMed]

5. Chiadroni, E.; Bacci, A.; Bellaveglia, M.; Boscolo, M.; Castellano, M.; Cultrera, L.; Di Pirro, G.; Ferrario, M.; Ficcadenti, L.; Filippetto, D.; et al. The SPARC linear accelerator based terahertz source. Appl. Phys. Lett. 2013, 102, 094101. [CrossRef]

6. Wu, Z.; Adli, E.; Fisher, A.; Loos, H.; Hogan, M. First Measurements of the FACET Coherent Terahertz Radiation Source. In Proceedings of the 3rd International Particle Accelerator Conference (IPAC2012), New Orleans, LA, USA, 20-25 May 2012.

7. van Tilborg, J.; Schroeder, C.B.; Tóth, C.; Geddes, C.G.R.; Esarey, E.; Leemans, W.P. Single-shot spatiotemporal measurements of high-field terahertz pulses. Opt. Lett. 2007, 32, 313-315. [CrossRef] [PubMed]

8. Neumann, J.G.; Fiorito, R.B.; O'Shea, P.G.; Loos, H.; Sheehy, B.; Shen, Y.; Zu, W. Terahertz laser modulation of electron beams. J. Appl. Phys. 2009, 105, 053304. [CrossRef]

9. Shen, Y.; Yang, X.; Carr, G.L.; Hidaka, Y.; Murphy, J.B.; Wang, X. Tunable Few-Cycle and Multicycle Coherent Terahertz Radiation from Relativistic Electrons. Phys. Rev. Lett. 2011, 107, 204801. [CrossRef]

10. Chiadroni, E.; Bellaveglia, M.; Calvani, P.; Castellano, M.; Catani, L.; Cianchi, A.; Di Pirro, G.; Ferrario, M.; Gatti, G.; Limaj, O.; et al. Characterization of the THz radiation source at the Frascati linear accelerator. Rev. Sci. Instrum. 2013, 84, 022703. [CrossRef]

11. Giorgianni, F.; Anania, M.P.; Bellaveglia, M.; Biagioni, A.; Chiadroni, E.; Cianchi, A.; Daniele, M.; Del Franco, M.; Di Giovenale, D.; Di Pirro, G.; et al. Tailoring of Highly Intense THz Radiation Through High Brightness Electron Beams Longitudinal Manipulation. Appl. Sci. 2016, 6, 56. [CrossRef] 
12. Anderson, S.G.; Musumeci, P.; Rosenzweig, J.B.; Brown, W.J.; England, R.J.; Ferrario, M.; Jacob, J.S.; Thompson, M.C.; Travish, G.; Tremaine, A.M.; et al. Velocity bunching of high-brightness electron beams. Phys. Rev. ST Accel. Beams 2005, 8, 014401. [CrossRef]

13. Piot, P.; Sun, Y.-E.; Maxwell, T.J.; Ruan, J.; Lumpkin, A.H.; Rihaoui, M.M.; Thurman-Keup, R. Observation of Coherently-Enhanced Tunable Narrow-Band Terahertz Transition Radiation from a Relativistic Sub-Picosecond Electron Bunch Train. Appl. Phys. Lett. 2011, 98, 261501. [CrossRef]

14. Bocek, D.; Hernandez, M.; Kung, P.; Lihn, H.C.; Settakorn, C.; Wiedemann, H. Observation of coherent undulator radiation from sub?picosecond electron pulses. AIP Conf. 1996, 367, 381-390. [CrossRef]

15. Zhang, J.; Deng, H.; Lin, X.; Dai, D.; Sun, Q.; Lu, S.; Yu, T.; Zhao, H.; Yang, H.; Dai, Z. Tunable few-cycle coherent terahertz radiation with watt-level power from relativistic femtosecond electron beam. Nucl. Instrum. Methods Phys. Res. A 2012, 693, 23-25. [CrossRef]

16. Green, B.; Kovalev, S.; Asgekar, V.; Geloni, G.; Lehnert, U.; Golz, T.; Kuntzsch, M.; Bauer, C.; Hauser, J.; Voigtlaender, J.; et al. High-Field High-Repetition-Rate Sources for the Coherent THz Control of Matter. Sci. Rep. 2016, 6, 22256. [CrossRef]

17. Wen, X.; Huang, S.; Lin, L.; Wang, F.; Zhu, F.; Feng, L.; Yang, L.; Wang, Z.; Fan, P.; Hao, J.; et al. Superradiant $\mathrm{THz}$ undulator radiation source based on a superconducting photo-injector. Nucl. Instrum. Meth. Phys. Res. A 2016, 820, 75-79. [CrossRef]

18. Kashiwagi, S.; Abe, T.; Saito, H.; Hinode, F.; Kanomata, K.; Miura, S.; Muto, T.; Nagasawa, I.; Nanbu, K.; Ninomiya, S.; et al. Observation of coherent undulator radiation in THz region. Infrared Phys. Technol. 2018, 93, 335-339. [CrossRef]

19. Krainara, S.; Zen, H.; Chatani, S.; Kii, T.; Ohgaki, H. Properties of THz coherent undulator radiation generated from a compact accelerator source at Kyoto University. Rev. Sci. Instrum. 2019, 90, 103307. [CrossRef]

20. Ferrario, M.; Alesini, D.; Anania, M.; Bacci, A.; Bellaveglia, M.; Bogdanov, O.; Boni, R.; Castellano, M.; Chiadroni, E.; Cianchi, A.; et al. SPARC_LAB present and future. Nucl. Instrum. Meth. Phys. Res. B 2013, 309, 183-188. [CrossRef]

21. Ginzburg, V.L.; Frank, I.M. Radiation of a uniformly moving electron due to its transition from one medium into another. J. Phys. USSR 1945, 9, 353-362.

22. Chiadroni, E. Bunch Length Characterization at the TTF VUV-FEL. Ph.D. Thesis, Tor Vergata University of Rome, Rome, Italy, 2006; TESLA-FEL Report No. 2006-09.

23. Serafini, L.; Ferrario, M. Velocity bunching in photo-injectors. AIP Conf. Proc. 2001, 581, 87-106.

24. Marchetti, B.; Bacci, A.; Chiadroni, E.; Cianchi, A.; Ferrario, M.; Mostacci, A.; Pompili, R.; Ronsivalle, C.; Spataro, B.; Zagorodnov, I.; et al. Novel schemes for the optimization of the SPARC narrow band THz source. Rev. Sci. Instrum. 2015, 86, 073301. [CrossRef]

25. Ferrario, M.; Alesini, D.; Bacci, A.; Bellaveglia, M.; Boni, R.; Boscolo, M.; Calvani, P.; Castellano, M.; Chiadroni, E.; Cianchi, A.; et al. Laser comb with velocity bunching: Preliminary results at SPARC. Nucl. Instr. Methods Phys. Res. Sect. A Accel. Spectrom. Detect. Assoc. Equip. 2011, 637, S43-S46. [CrossRef]

26. Villa, F.; Anania, M.P.; Bellaveglia, M.; Bisesto, F.; Chiadroni, E.; Cianchi, A.; Curcio, A.; Galletti, M.; Di Giovenale, D.; Di Pirro, G.; et al. Laser pulse shaping for high gradient accelerators. Nucl. Instrum. Methods Phys. Res. Sect. A 2016, 829, 446-451. [CrossRef]

27. Mostacci, A.; Alesini, D.; Antici, P.; Bacci, A.; Bellaveglia, M.; Boni, R.; Castellano, M.; Chiadroni, E.; Cianchi, A.; Di Pirro, G.; et al. Advanced beam manipulation techniques at SPARC. In Proceedings of the IPAC2011, THYB01, San Sebastian, Spain, 4-9 September 2011.

28. Martin, D.H.; Puplett, E. Polarized interferometric spectrometry for the millimeter and sub-millimeter spectrum. Infrared Phys. 1970, 10, 105-109. [CrossRef]

29. All THz band-pass filters but the $5 \mathrm{THz}$ one are produced by Tydex, whose part number designation is: BPF0.3-47, BPF0.5-47, BPF1.0-47, BPF3.0-47, with 47 the clear aperture in $\mathrm{mm}$; the $5 \mathrm{THz}$ band-pass filter is manufactured by Lake Shore Cryotronics, Inc. (https:/ /www.lakeshore.com/about-us / press-releases/2007/11/05/new-far-infrared-thz-band-pass-filters), and has been also characterized by measuring with a Michelson interferometer the globar spectrum at $5 \mathrm{THz}$.

30. Castellano, M.; Cianchi, A.; Orlandi, G.; Verzilov, V.A. Effects of diffraction and target finite size on coherent transition radiation spectra in bunch length measurements. Nucl. Instrum. Methods Phys. Res. A 1999, 435, 297-307. [CrossRef] 
31. Stojanovic, N.; Drescher, M. Accelerator- and laser-based sources of high-field terahertz pulses. J. Phys. B At. Mol. Opt. Phys. 2013, 46, 192001. [CrossRef]

32. Bass, M.; Franken, P.A.; Ward, J.F.; Weinreich, G. Optical Rectification. Phys. Rev. Lett. 1962, 9, 446. [CrossRef]

33. Vicario, C.; Jazbinsek, M.; Ovchinnikov, A.V.; Chefonov, O.V.; Ashitkov, S.I.; Agranat, M.B.; Hauri, C.P. High Efficiency THz Generation in DSTMS, DAST and OH1 pumped by Cr:forsterite laser. Opt. Express 2015, 23, 4573-4580. [CrossRef] [PubMed]

34. Giorgianni, F.; Chiadroni, E.; Rovere, A.; Cestelli-Guidi, M.; Perucchi, A.; Bellaveglia, M.; Castellano, M.; Di Giovenale, D.; Di Pirro, G.; Ferrario, M.; et al. Strong nonlinear terahertz response induced by Dirac surface states in Bi2Se3 topological insulator. Nat. Commun. 2016, 7, 11421. [CrossRef] [PubMed]

(C) 2020 by the authors. Licensee MDPI, Basel, Switzerland. This article is an open access article distributed under the terms and conditions of the Creative Commons Attribution (CC BY) license (http://creativecommons.org/licenses/by/4.0/). 